\title{
Low Gravity Natural Convection and Pool Boiling Predictions
}

\author{
Ashley Milligan ${ }^{1}$ \\ University of Memphis, Memphis, Tennessee, 38111, United States
}

\begin{abstract}
Cryogenic fluids are used in various fields, such as biomedical technology, food transportation, and aerospace. These fluids have optimal properties as an energy-dense fuel for long-term, large payload space flight. Much of the resulting research in long-duration cryogenic fluid storage has focused on zero-boil-off tank pressure control in low gravity environments. The high cost of testing these systems has created a need for modeling these environments. Simulations of tank pressure control require accurate models of evaporation and condensation phase change. Recent advancements in commercial fluid dynamics simulation software offer potential to better simulate evaporation/condensation phase change. The goal of the present work is to use commercial software to simulate pool boiling of cryogenic propellants in low gravity. The volume of fluid approach with the Lee phase change model, which predicts heat and mass transfer at the liquid vapor interface, has been recently added to the commercial software. Accurately modeling natural convection is a key component of simulating pool boiling. Results from natural convection simulations at low gravity levels are presented and are comparable to experimental data and previously published numerical simulations. Future plans for nucleate pool boiling simulations are also presented.
\end{abstract}

\section{Nomenclature}

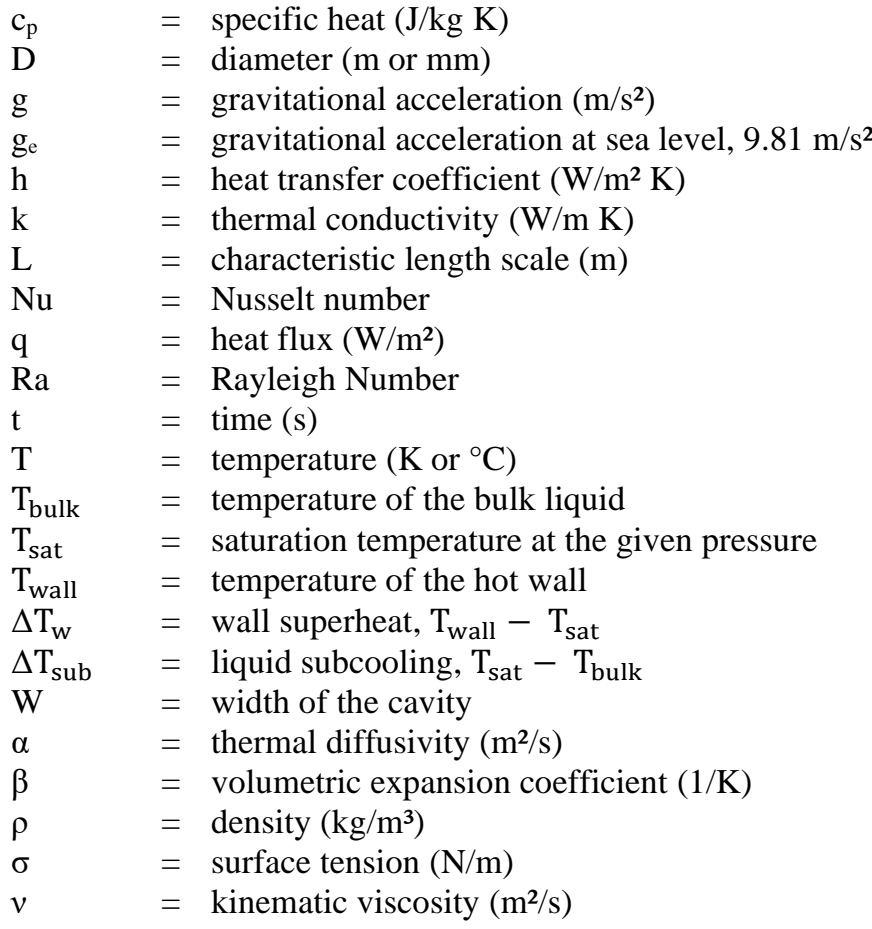

\footnotetext{
${ }^{1}$ Graduate Researcher, Department of Mechanical Engineering, AIAA Student Member
} 


\section{Introduction}

Systems involving substances at temperatures below $-150^{\circ} \mathrm{C}\left(-238^{\circ} \mathrm{F}\right)$ are referred to as cryogenics. There are many cryogenic fluids that are liquids at these extreme temperatures, such as liquid hydrogen, oxygen, and nitrogen. Many industries utilize cryogenic fluids for a variety of purposes, such as food storage, chemical reactors, and rocket propellant [1].

There are many uses for cryogenics specifically in space applications. While using fluids such as liquid hydrogen or liquid oxygen for rocket propellants is the most well-known application in this industry, it is becoming more common to see research geared towards the use of cryogenics in low temperature storage and life support systems. With the increasing interest in deep space travel and long-term manned missions, cryogenic fuel storage has become an interesting research field.

Effective tank design is crucial to prevent propellant loss due to boiling over long-term missions. Design and testing of large-scale cryogenic fluid storage tanks in low gravity would be exceedingly expensive and time consuming. Thus, further design and development of cryogenic propellant management systems necessitates accurate models of cryogenic propellant fluid-thermal behavior in low gravity. In particular, a robust simulation of low gravity boiling of cryogenic fluids is needed to further develop a useful large-scale zero-boil-off tank. Researchers have been developing correlations and numerical simulations for pool nucleate boiling since the 1950s, and many of these still differ greatly from one another [2]. Multiple theoretical and computational models of varying complexity have been developed to simulate tank pressurization behavior, but are inconsistent when compared to experimental data [3]. Simulations of tank pressure control require accurate physics models, including evaporation and condensation phase change in low gravity. Recently, numerical simulations have been created that can simulate natural convection, nucleate pool boiling, and bubble growth and departure in microgravity with great accuracy compared to experiments [4-6]. These simulations are often complex and require a great knowledge of programming and computational fluid dynamics (CFD). However, recently developed models of evaporation and condensation have been added to commercial CFD software that may enable accurate simulation of low gravity natural convection and pool boiling [79]. This works aims to assess whether these recently added evaporation and condensation models can be used to accurately model pool boiling in low gravity. Simulation results for natural convection in low gravity are presented and compared to experiment data to validate the model. Further, plans are presented to validate the ability of the model to predict nucleate pool boiling in low gravity.

\section{Background}

\section{A. Low Gravity Liquid Storage}

Cryogenic propellants are the leading candidate for propulsion systems for long duration manned space missions. Cryogenic propellants have a higher specific impulse than solid propellants, which means that more energy is comparatively produced per pound of propellant. One issue with storing cryogens for long durations is boil off and subsequent loss of stored propellant. Improving storage technologies will lead to decreased propellant loss $[1,10]$.

Much of the research in long-duration cryogenic fluid storage has focused on zero-boil-off (ZBO) tank pressure control in low gravity environments [10]. Research in improved tank design often focuses on active and passive technologies to decrease boiling. Active technology in this scenario is most often the use of an active cryogenic refrigerator (cryocooler) [11]. Active systems require power which is limited on spacecraft. Passive technologies consist of technologies such as vapor-cooled shields, sun shades, and tank insulation [12]. While tank designs are improving and some studies have shown that zero boil-off tanks can be achieved deeper in space, the ability to validate each new improvement is still extremely expensive and time intensive..

\section{B. Experimental Studies}

Experiments regarding low-gravity boiling heat transfer were first performed in the 1960's. Siegel and Keshock [13] conducted NASA-sponsored experiments which utilized drop towers and lasted only a few seconds. The studies involved electrically heated wires in freefall and evaluated bubble growth and departure. Distilled water, liquid nitrogen, and alcohol were all studied. The liquids were saturated and on horizontal, heated wires and ribbons. The boiling curve did not appear to be greatly affected by change in gravity level. Longer duration low gravity experiments involving magnetic particles were carried out by Papell and Faber [14]. They were able to achieve a steady-state boiling curve, which showed a decrease in wall superheat when gravity changed from earth gravity to microgravity.

Pool boiling experiments were first performed on the International Space Station (ISS) by Merte, Lee, and Keller $[15,16]$ in the 1990's aboard the space shuttle. The fluid analyzed was the fluorocarbon R-113. The experiments 
studied pool boiling at steady-state by varying the heat flux and liquid subcooling. Bubble formation and growth was observed in many cases, and pool boiling curves for R-113 were created for two levels of subcooling.

Raj, Kim, and McQuillen performed experiments in the Boiling Experimental Facility (BXF) on the ISS during March - May of 2011 [17]. The Microheater Array Boiling Experiment (MABE) was one of two experiments within the BXF. The experiment evaluated many boiling characteristics in low gravity, such as the onset of nucleate boiling and the effect of subcooling and pressure. The researchers found that as pressure increased, the superheat needed for nucleate boiling to begin decrease, but in low gravity, nucleate boiling occurred, and smaller superheats were needed compared to tests performed in earth gravity. Heat flux values at lower superheats are shown to be greater than they would be in earth gravity environments. They also found that heat transfer rates were proportional to liquid subcooling and pressure.

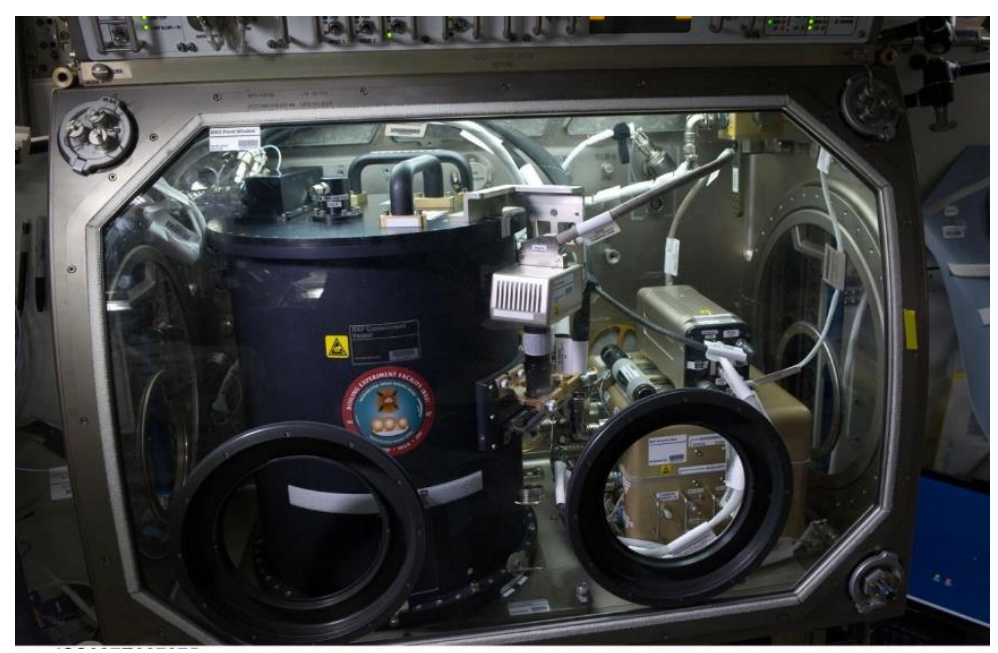

Fig. 1 The Boiling Experimental Facility on the ISS [4].

The second experiment housed in the BXF was the Nucleate Pool Boiling Experiment (NPBX) by Warrier et al. [4]. In this experiment, the test chamber was filled with perfluoro-n-hexane and maintained at a constant pressure with the use of bellows. The fluid was initially at a constant bulk temperature, and an $89.5 \mathrm{~mm}$ aluminum wafer was heated from the underside. Experiments were performed to obtain data for natural convection, nucleate pool boiling, and bubble dynamics. The results compared favorably with previous experiments and numerical correlations. The data from this experiment has been used to create numerical models and is discussed in greater detail in later sections.

\section{Computational Studies}

Experimental data from the two experiments from the BXF were used to verify models created by researchers from the two groups. The model by Raj et al. predicted the low gravity experimental data accurately. Models by Aktinol and Dhir [5] were able to recreate the bubble formation and growth in low gravity.

Studies have assessed the validity of evaporation models for cryogenic liquid spills at normal earth gravity compared to experimental data. Midha et al. found that the CFD tool FLACS has predicted hydrogen gas dispersion from liquid hydrogen spills [18]. Nawaz et al. analyzed six evaporation models, and found that they all over-predicted the vaporized liquid mass [19]. Multiple theoretical and computational models of varying complexity have been developed to simulate tank pressurization behavior, but are inconsistent compared to one other and experimental data [3].

Recently, models for evaporation and condensation phase change with nucleate boiling were introduced in commercial computational fluid dynamics (CFD) software [7]. Sun et al. have used user-defined functions (UDFs) to simulate phase change using a VOF model [16]. The volume-of-fluid (VOF) multiphase model solves one set of momentum equations and tracks the volume fraction of each fluid within the domain, and is utilized in many commercial software packages [20]. In the CFD software studied, the VOF model uses the Lee Model for evaporation and condensation to predict boiling activity [21]. 


\section{Low Gravity Natural Convection Validation Case I: The Stefan Problem}

To validate the ability for the commercial software to predict and model low gravity pool boiling, it is first necessary to confirm the ability of the software to accurately simulate natural convection in various gravity levels. This case utilizes the Boussinesq density model and analyzes laminar and turbulent models for several Rayleigh numbers. The results are compared to published mathematical models, numerical simulations, and experiments.

$$
\begin{gathered}
\mathrm{Nu}=\frac{h k}{L} \\
h=\frac{q}{T_{\text {wall }}-T_{\text {bulk }}} \\
\mathrm{Ra}=\frac{g \beta\left(T_{\text {wall }}-T_{\text {bulk }}\right) L^{3}}{v \alpha}
\end{gathered}
$$

\section{A. Background}

A common type of boundary value problems analyzed in phase change mathematics are Stefan problems. The type of Stefan problems evaluated in this work involve differentially heated walls on either side of the domain. Experiments have been performed in order to validate numerical models and found that it is often simpler and more efficient to numerically compute state characteristics than determine the same characteristics experimentally for low Rayleigh numbers [22]. Numerical and computational methods have been utilized to accurately predict solutions to natural convection cases for a range of Rayleigh numbers [23,24]. Many of these studies have led to Nusselt and Rayleigh number correlations that will be compared to the results presented herein.

\section{B. Computational Setup}

The model consists of a square domain with a non-uniform mesh that is more refined as it approaches each wall. The Rayleigh number was altered by changing the scale of the model as well as the gravity level. The scale of the model is defined by a value $\mathrm{W}$, which is the width of the square domain. Eight different domain sizes are analyzed. For every case, the hot and cold walls are maintained at constant temperatures of $303 \mathrm{~K}$ and $283 \mathrm{~K}$, respectively. The two remaining walls are adiabatic, and the interior fluid is initialized at $293 \mathrm{~K}$. The boundary conditions and general geometry are shown in Figure 2. Continuity, momentum, and energy are computed using a segregated solver [8]. The fluid modeled is air at atmospheric pressure, with the Boussinesq approximation [25] applied for density, and all other properties assumed to be constant.

A steady state solution is found for varying values of $\mathrm{W}$ to create a range of laminar Rayleigh numbers less than $10^{6}$ for both the earth gravity and low gravity cases. In the low gravity cases, gravity is assumed to be approximately $\mathrm{g} / \mathrm{g}_{\mathrm{e}}=10^{-7}$.

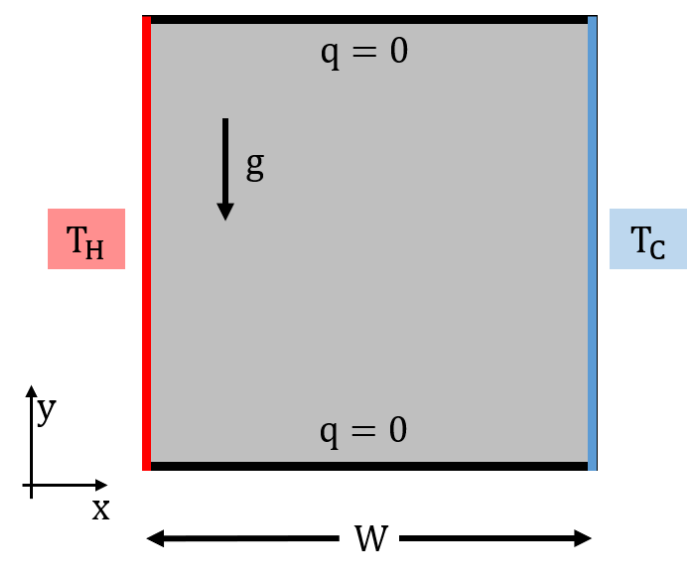

Fig. 2 Natural Convection in a 2-D Square boundary conditions. 


\section{Results}

The results for Stefan cases using earth's gravity compare favorably with laminar correlations found by Barakos et al. [23] and Markatos and Pericleous [24]. The low gravity cases show little variation in Nusselt number for the two smallest Rayleigh number cases but begin to follow the same trend as the earth gravity cases starting in the range of $\mathrm{Ra}=10^{2}$, which is the range of the experimental data from Warrier et al. [4]. The characteristic length stale, $\mathrm{L}$, in this case is the width of the domain, W. Nusselt Number vs. Rayleigh Number correlations are plotted in Figure 3.

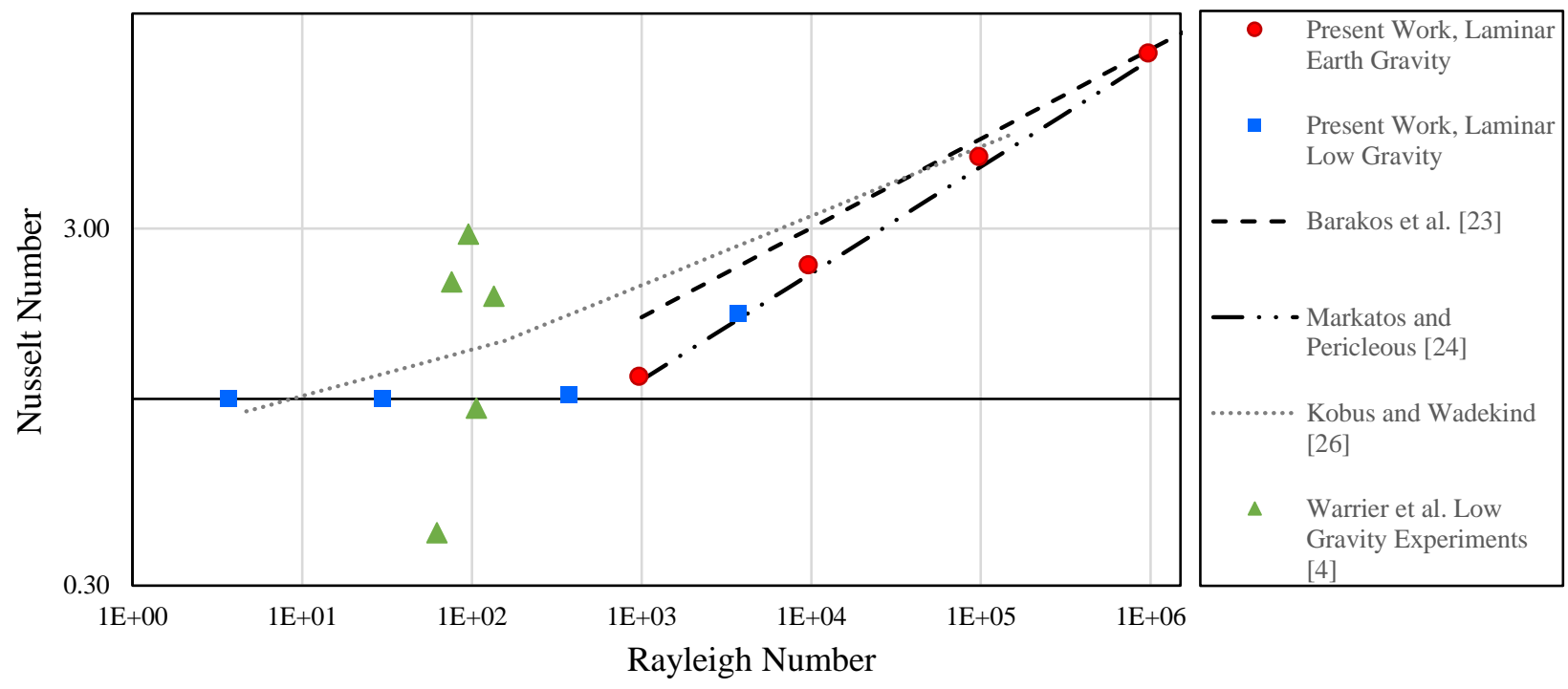

Fig. 3 Dimensionless number correlations for laminar flow.

From these results, it can be assumed that the commercial software is accurate for Rayleigh numbers less than $10^{6}$ for earth gravity cases, and for $10^{2}<\mathrm{Ra}<10^{6}$ for low gravity cases.

\section{Low Gravity Natural Convection Validation Case II: Nucleate Pool Boiling Experiments (NPBX)}

To model nucleate pool boiling, it is again necessary to accurately predict natural convection flow characteristics. These models were created using an axisymmetric domain to simulate experimental results from the Nucleate Pool Boiling Experiments (NPBX). The flow is assumed to be laminar, and the hot plate is at a constant heat flux. Two different heat flux values are evaluated at the same liquid subcooling, and the simulations are run until the Nusselt number has converged. Nusselt and Rayleigh number correlations are presented, and a mesh study was conducted.

\section{A. Background}

Experiments have been performed on the International Space Station to better increase knowledge of natural convection and nucleate pool boiling in microgravity. These results are useful for calibrating, testing, and validating numerical simulations of low gravity boiling [4,5]. In this section, natural convection simulations based off experiments will be discussed.

The Nucleate Pool Boiling Experiments were performed on the International Space Station inside the Boiling Experiment Facility (BXF) during March - May of 2011 on Space Shuttle Mission STS-133. The BXF contained a diamond turned aluminum wafer that was $1 \mathrm{~mm}$ thick and $89.5 \mathrm{~mm}$ in diameter. This wafer was the boiling surface for the experiment, and was heated from the backside with strain gage heaters. The wafer was placed inside the test chamber, which contained bellows to maintain a constant pressure and pumps to regulate the initial temperature before starting each test. The test chamber was filled with perfluoro-n-hexane and degassed routinely. [4] 


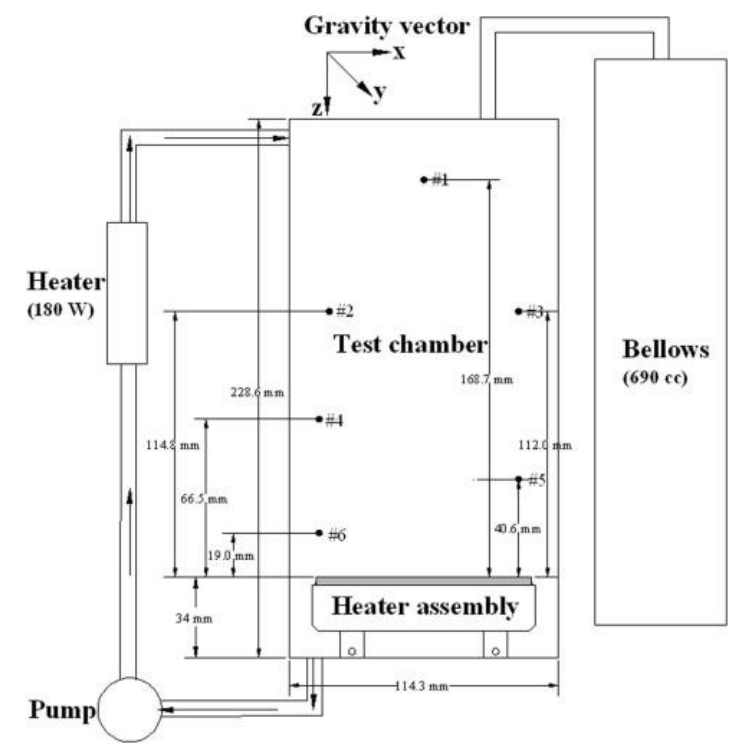

Fig. 4 The Nucleate Pool Boiling Experiment test chamber [4].

Experiments were conducted to investigate the characteristics of natural convection in microgravity. Data from these experiments show a large range in Nusselt numbers, but are close to correlations by Kobus and Wadekind [26]. However, it is estimated that the time required to reach steady state for the setup is about 24,000 s based on the critical Rayleigh number and conduction layer thickness, whereas the experiments conducted on the ISS never lasted more than $1,800 \mathrm{~s}$.

Nucleate pool boiling experiments were also conducted, and while the nucleate boiling data will not be analyzed in this section, data for the natural convection range of the boiling curve are presented, as well as data for the pressure, heat flux, and temperatures needed to create a comparable model. Aktinol and Dhir implemented numerical models using data from the NPBX experiments. While most of their analysis involved nucleate boiling and bubble dynamics, one case presented predicts natural convection behavior. Assumptions and material properties used by Aktinol are described and used to create the model presented in this section. [5]

\section{B. Computational Setup}

For the natural convection simulations, a rectangular mesh is created. This mesh is $44.75 \mathrm{~mm}$ by $225.6 \mathrm{~mm}$ and is axisymmetric as described in Figure 6. There is also a $10 \mathrm{~mm}$ radius section to represent the bellows in the NPBX case. The model utilizes a transient solver with a time step of $1 \mathrm{~s}$. This time step was chosen after a time step study was conducted and showed little variation. A laminar viscosity model is applied since the Rayleigh number is expected to be within the laminar range. The fluid modeled inside the domain is perfluoro-n-hexane ( $\mathrm{PnH})$. The Boussinesq density approximation is used, and all material properties are assumed to be constant. Material properties for PnH are listed in Table 1. A constant heat flux is applied at the hot wall, and an opposite heat flux adjusted for the area is applied at the outlet, which is modeled as a wall since the pressure is not expected to change since there will be no phase change. The remaining walls are all adiabatic, and an axis of symmetry exists at $y=0$. Since the axis represents the vertical center of the boiling container, gravity is applied in the negative $\mathrm{x}$ direction. 


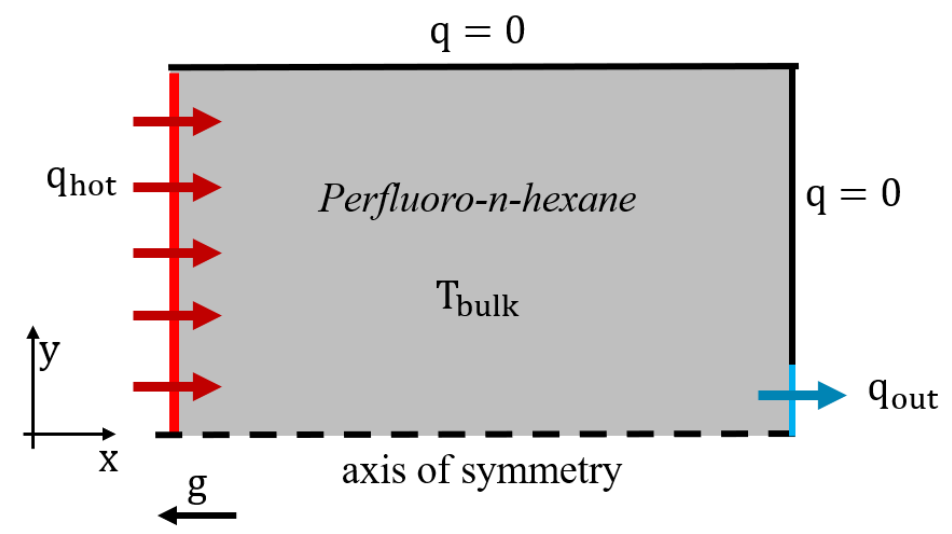

Fig. 5 Domain setup and boundary conditions for the numerical model.

Nusselt number and Rayleigh number are calculated using the temperature difference between the hot wall and the average bulk fluid temperature, which is roughly equal to the initial temperature. The characteristic length, $\mathrm{L}$, used is equal to the diameter of the heater divided by 4 . Each case initially ran for a flow time of $1800 \mathrm{~s}$ to compare the result to the results from the NPBX if the fluid did not actually reach steady state. The model is then executed until the Nusselt number converges to get a more accurate comparison to established correlations by Kobus and Wadekind [26]. For each heat flux value, a mesh study has also been performed. Three different uniform meshes of differing cell sizes were studied.

\begin{tabular}{|c|c|c|}
\hline \multirow{2}{*}{ Density, $\rho\left(\mathrm{kg} / \mathrm{m}^{3}\right)$} & Liquid & 1592 \\
\cline { 2 - 3 } & Vapor & 13.4 \\
\hline \multirow{2}{*}{ Specific Heat, $\mathrm{c}_{\mathrm{p}}(\mathrm{J} / \mathrm{kg} \mathrm{K})$} & Liquid & 1102 \\
\cline { 2 - 3 } & Vapor & 236 \\
\hline Thermal Conductivity, $\mathrm{k}(\mathrm{W} / \mathrm{m} \mathrm{K})$ & Liquid & 0.0537 \\
\cline { 2 - 3 } & Vapor & 0.00260 \\
\hline Viscosity, $\mu(\mathrm{kg} / \mathrm{m} \mathrm{s})$ & Liquid & $11.6 \times 10^{-6}$ \\
\cline { 2 - 3 } & Vapor & 0.0016 \\
\hline Thermal Expansion Coefficient, $\beta$ & Liquid \\
\hline Standard State Enthalpy $(\mathrm{J} / \mathrm{kgmol})$ & \multicolumn{2}{|c|}{338} \\
\hline Molecular Weight $(\mathrm{kg} / \mathrm{kmol})$ & \multicolumn{2}{|c|}{$3.20 \times 10^{7}$} \\
\hline
\end{tabular}

Table 1 Material properties for perfluoro-n-hexane in the liquid and gaseous state [5].

\section{Results}

The results from the simulations are compared to results from Warrier et al. [4] and numerical correlations by Kobus and Wadekind [26]. In Figure 7, two sets of results are presented: one set for a flow time of $1800 \mathrm{~s}$, and the second after quasi-steady state conditions were achieved, which occurred around a flow time of 120,000 s. The 1800 $\mathrm{s}$ results do not differ with Rayleigh number, and are slightly larger in magnitude than the highest Nusselt number found from the experiments by Warrier et al. The quasi-steady results are much more accurate when it comes to Nusselt and Rayleigh number correlations, but the Rayleigh numbers are all slightly larger than the experiments. This is likely due to either a larger temperature difference between the hot wall and the bulk fluid caused by a longer heating time, difference in the magnitude of gravitational acceleration used to calculate Rayleigh number, or a combination of the two factors. 


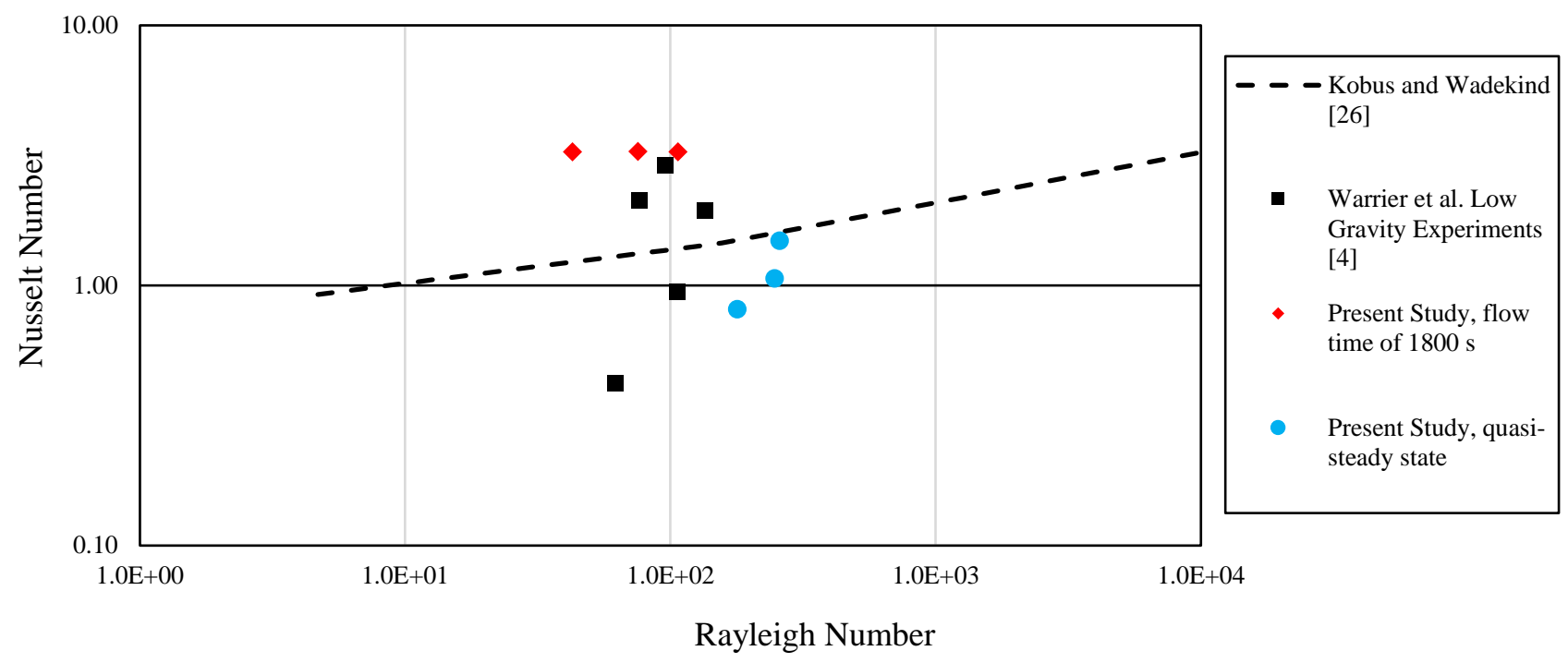

Fig. 6 Low gravity dimensionless number correlations

Velocity contours of the flow, shown in Figure 8, demonstrate that buoyancy-driven natural convection flow is occurring in the domain. The maximum magnitude of the velocity in quasi-steady flow is $2.76 \times 10^{-5} \mathrm{~m} / \mathrm{s}$. Ultimately, this shows that the commercial software can predict circulation due to natural convection is happening in the fluid in a low gravity condition.
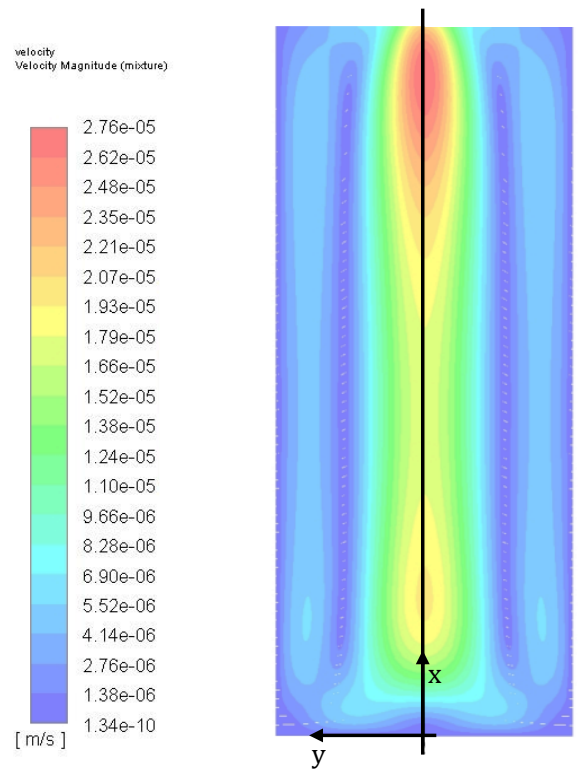

Fig. 7 Velocity vectors for the domain rotated 90 degrees with the hot plate at the bottom of the image.

A mesh study was performed for the cases to determine if the solution was mesh-independent. Three different mesh cell sizes were analyzed. From the analysis, it can be seen that for the first 3,500 s of flow time, the results produced using each mesh are indistinguishable. However, around this time the results obtained using the less dense meshes show an increase in Nusselt number. The results obtained using the denser mesh show an increase in Nusselt number around 4,500 s. Fluctiations in the Nusselt number occur, and the solutions are not equivalent at each time step, but have a similar amplitude. For all meshes, the Nusselt number for each mesh converges at approximately $120,000 \mathrm{~s}$. 


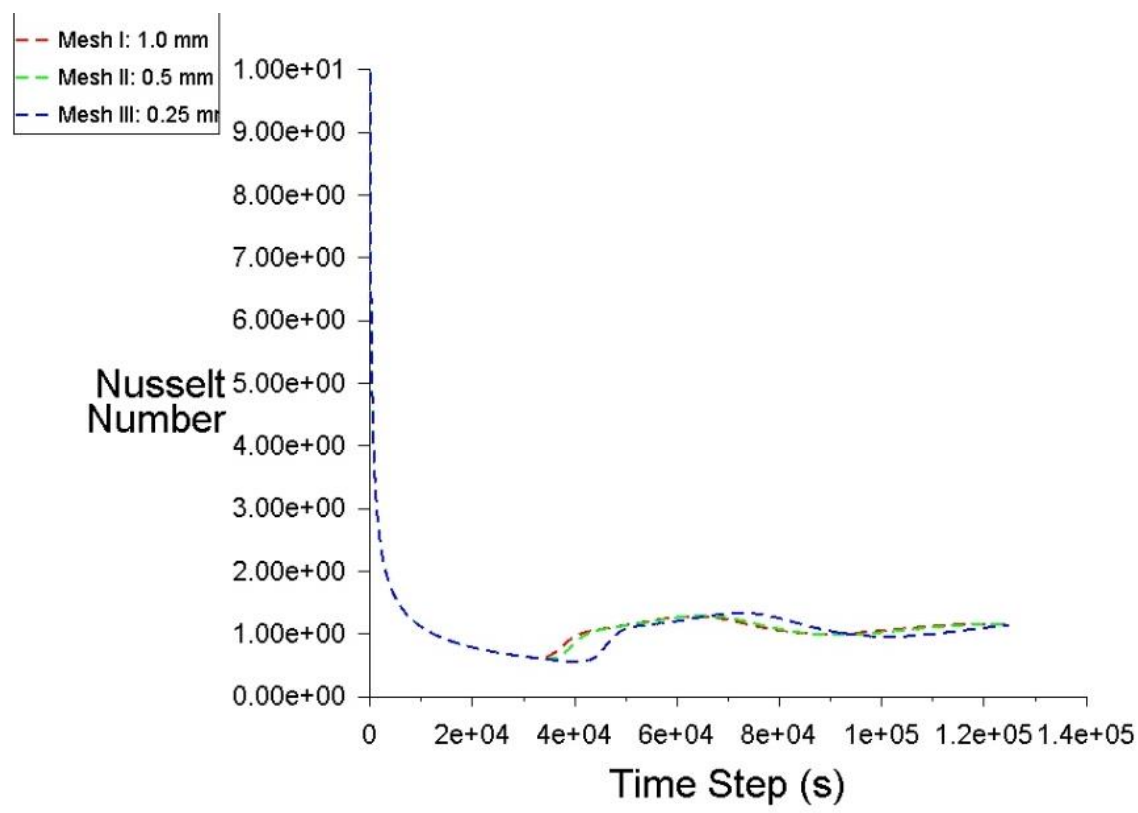

Fig. 8 Nusselt number as a function of time for various mesh sizes.

\section{Future Work: Nucleate Boiling}

\section{A. Background}

In the NPBX experiments, the primary aims of the experiments were to study nucleate boiling and bubble dynamics. Using data from these experiments, Dhir and other researchers have successfully simulated the experiment results, however, these models have not been proven for a broader variety of geometries and conditions. During the nucleate boiling portions of the NPBX, the wafer temperature was increased until nucleation occurred, then the wafer was reduced to the initial temperature until quasi steady-state conditions were present. After this, the wafer temperature was increased step by step to capture data for along the boiling curve.

The numerical model by Aktinol and Dhir [6] uses an iterative procedure and a level set method to track the liquidvapor interface. The model is two dimensional and axisymmetric, and couples the solid substrate heater. Different simulations are performed with different initial conditions. Multiple cases are described, and all consist of a set pressure, liquid subcooling, and wall superheat. The NPBX cases will be adopted to validate the commercial model for use in predicting low gravity nucleate pool boiling.

\section{B. Computational Setup}

The nucleate pool boiling model available in the commercial software is currently being setup as described in section V-B. A 3-dimensional model will be required to simulate nucleate pool boiling. The current approach is to model natural convection with a constant surface heat flux until the wall temperature reaches the saturation temperature of the fluid. After this, the multiphase evaporation and condensation model will be incorporated. This model uses the Volume of Fluid approach with the Lee Phase Change model. Once the multiphase model is turned on, a few different approaches will be analyzed. First, a constant wall temperature above the saturation temperature will be applied to the heated wall until it appears that quasi steady conditions have been reached. Then, like in the NPBX, the temperature will be increased incrementally while recording values for wall heat flux among other things.

A second approach is to let the heat flux continue to increase until phase change begins to occur, after which a constant wall temperature will be set, and the same steps as above will be followed. The goal of this is to predict the heat flux at each wall superheat and compare the results to those presented by Warrier et al. [4]. 


\section{Conclusion}

Natural convection in microgravity has been predicted using a commercial software with no user-defined functions and has shown to be comparable to experimental data and numerical correlations. With this step of the boiling curve complete, the software will be used to simulate low gravity nucleate pool boiling. Results from the simulation will be compared to available experimental data to validate the commercial model. Once validated the commercial software will be used to simulate other low gravity experiments to further demonstrate its capability. Simulations can then be performed to aid in the design of cryogenic propellant management systems for space applications.

\section{Acknowledgements}

The author would like to thank the Herff College of Engineering Graduate Fellowship and the Department of

Mechanical Engineering for sponsoring and funding this research.

\section{References}

[1] Salerno, L. J., and Kittel, P. "Cryogenics and the Human Exploration of Mars." Cryogenics, Vol. 39, No. 4, 1999, pp. 381-388. https://doi.org/10.1016/S0011-2275(99)00043-0.

[2] Dhir, V. K. "Mechanistic Prediction of Nucleate Boiling Heat Transfer-Achievable or a Hopeless Task?" Journal of Heat Transfer, Vol. 128, No. 1, 2006, pp. 1-12. https://doi.org/10.1115/1.2136366.

[3] Barsi, S., and Kassemi, M. "Investigation of Tank Pressurization and Pressure Control-Part II: Numerical Modeling." Journal of Thermal Science and Engineering Applications, Vol. 5, No. 4, 2013, p. 041006. https://doi.org/10.1115/1.4023892.

[4] Warrier, G. R., Dhir, V. K., and Chao, D. F. "Nucleate Pool Boiling EXperiment (NPBX) in Microgravity: International Space Station." International Journal of Heat and Mass Transfer, Vol. 83, 2015, pp. 781-798. https://doi.org/10.1016/j.ijheatmasstransfer.2014.12.054.

[5] Aktinol, E. Numerical Simulations of Bubble Dynamics and Heat Transfer in Pool Boiling--Including the Effects of Conjugate Conduction, Level of Gravity, and Noncondensable Gas Dissolved in the Liquid. UCLA, 2014.

[6] Aktinol, E., and Dhir, V. K. "Numerical Simulation of Nucleate Boiling Phenomenon Coupled with Thermal Response of the Solid." Microgravity Science and Technology, Vol. 24, No. 4, 2012, pp. 255-265. https://doi.org/10.1007/s12217-012-9308-7.

[7] ANSYS® Fluent 19.1. ANSYS, Inc.

[8] ANSYS® Fluent 19.1 User Guide. ANSYS, Inc.

[9] ANSYS® Fluent 19.1 Theory Guide. ANSYS, Inc.

[10] Hastings, L. J., Plachta, D. W., Salerno, L., and Kittel, P. "An Overview of NASA Efforts on Zero Boiloff Storage of Cryogenic Propellants." 2002, p. 8.

[11] Kittel, P., Salerno, L. J., and Plachta, D. W. Cryocoolers for Human and Robotic Missions to Mars. In Cryocoolers 10 (R. G. Ross, ed.), Springer US, Boston, MA, 2002, pp. 815-821.

[12] Plachta, D. W., Christie, R. J., Jurns, J. M., and Kittel, P. "Passive ZBO Storage of Liquid Hydrogen and Liquid Oxygen Applied to Space Science Mission Concepts." Cryogenics, Vol. 46, Nos. 2-3, 2006, pp. 89-97. https://doi.org/10.1016/j.cryogenics.2005.11.012.

[13] Siegel, R., and Keshock, E. G. "Effects of Reduced Gravity on Nucleate Boiling Bubble Dynamics in Saturated Water." AIChE Journal, Vol. 10, No. 4, 1964, pp. 509-517. https://doi.org/10.1002/aic.690100419.

[14] Papell, S. S., and Faber, O. C. Zero- and Reduced-Gravity Simulation on a Magnetic-Colloid Pool-Boiling System. National Aeronautics and Space Administration, 1966.

[15] Merte, Jr., H., Lee, H., and Robert Keller. Report on Pool Boiling Experiment Prototype Model Flown on STS47 (PBE-IA). Publication UM-MEAM-94-09. NASA, 1994, p. 265.

[16] Lee, H., Merte, Jr., H., and Chiaramonte, F. The Pool Boiling Curve in Microgravity. Presented at the 34th Aerospace Sciences Meeting and Exhibit, Reno,NV,U.S.A., 1996.

[17] Raj, R., Kim, J., and McQuillen, J. "Pool Boiling Heat Transfer on the International Space Station: Experimental Results and Model Verification." Journal of Heat Transfer, Vol. 134, No. 101504, 2012. https://doi.org/10.1115/1.4006846.

[18] Middha, P., Ichard, M., and Arntzen, B. J. "Validation of CFD Modelling of LH2 Spread and Evaporation against Large-Scale Spill Experiments." International Journal of Hydrogen Energy, Vol. 36, No. 3, 2011, pp. 2620-2627. https://doi.org/10.1016/j.ijhydene.2010.03.122. 
[19] Nawaz, W., Olewski, T., and Véchot, L. "Assessment and Validation of Evaporation Models for Cryogenic Liquids." Process Safety and Environmental Protection, Vol. 121, 2019, pp. 50-61. https://doi.org/10.1016/j.psep.2018.08.013.

[20] Welch, S. W. J., and Wilson, J. "A Volume of Fluid Based Method for Fluid Flows with Phase Change.” Journal of Computational Physics, Vol. 160, No. 2, 2000, pp. 662-682. https://doi.org/10.1006/jcph.2000.6481.

[21] Lee, W. H. "A Pressure Iteration Scheme for Two-Phase Flow Modeling." MATHEMATICAL MODELING, p. 25.

[22] MacGregor, R. K., and Emery, A. F. "Free Convection Through Vertical Plane Layers-Moderate and High Prandtl Number Fluids." Journal of Heat Transfer, Vol. 91, No. 3, 1969, pp. 391-401. https://doi.org/10.1115/1.3580194.

[23] Barakos, G., Mitsoulis, E., and Assimacopoulos, D. "Natural Convection Flow in a Square Cavity Revisited: Laminar and Turbulent Models with Wall Functions." International Journal for Numerical Methods in Fluids, Vol. 18, No. 7, 1994, pp. 695-719. https://doi.org/10.1002/fld.1650180705.

[24] Markatos, N. C., and Pericleous, K. A. "Laminar and Turbulent Natural Convection in an Enclosed Cavity." International Journal of Heat and Mass Transfer, Vol. 27, No. 5, 1984, pp. 755-772. https://doi.org/10.1016/0017-9310(84)90145-5.

[25] Boussinesq, J. Théorie de l'écoulement tourbillonnant et tumultueux des liquides dans les lits rectilignes a grande section. Paris, Gauthier-Villars et fils, 1897.

[26] Kobus, C. J., and Wedekind, G. L. "An Experimental Investigation into Natural Convection Heat Transfer from Horizontal Isothermal Circular Disks." International Journal of Heat and Mass Transfer, Vol. 44, No. 17, 2001, pp. 3381-3384. https://doi.org/10.1016/S0017-9310(00)00330-6. 